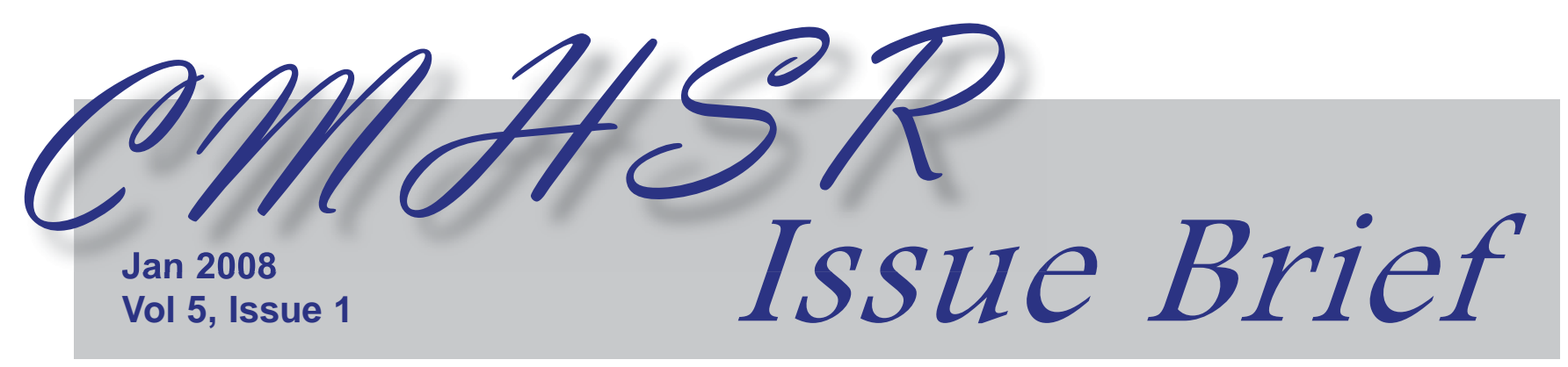

\title{
Adolescent Mental Health Gender Differences in Arrest Onset \& Risk
}

Maryann Davis, PhD, William H. Fisher, PhD, Steven Banks, PhD, Bernice Gershenson, MPH

$\mathrm{R}$ esearch conducted at CMHSR indicates that most males and almost half of females who services as an adolescent have an arrest by age 25 , with a majority of them having been arrested multiple times. ${ }^{1}$ However, at any given age, the likelihood of first arrest is low $(2-16 \%$ with a first arrest). Low risk generally precludes the application of primary prevention interventions, which would be applied to the whole population of adolescent public mental health system users, unless they are inexpensive and can be applied safely. Furthermore, primary prevention approaches would then include those adolescents who may pose no real risk of subsequent arrest. However, if there was sufficient information to easily identify adolescents at high risk and if there were approaches to help prevent or reduce offending, application of those approaches to the small number of well-targeted individuals would be highly beneficial.

\section{Arrest Patterns of Juveniles}

Using data from the Massachusetts Department of Mental Health's (DMH), and Massachusetts Juvenile and Criminal Courts' administrative databases, CMHSR researchers examined juvenile and criminal arrests in a statewide cohort of persons receiving intensive public adolescent mental health services. To identify arrests experienced between 7 and 24 years of age, researchers linked arrest records to the records of youths who received DMH adolescent case management services sometime in 1994-96 and who were born between 1976-79 (781 males, 738 females). Researchers compared arrest patterns of males and females at each age between 14 and 24 years (few were arrested before age 14), to determine whether there were developmental trends and gender differences.

\section{Results}

The highest risk of imminent arrest (i.e. arrest within the next year) was among those who have had a recent arrest. Once arrested, and depending on age, between $26-39 \%$ of females and $31-50 \%$ of males will be arrested again in the following year. Further, if that was not their first arrest, their risk of arrest the following year was between $29-50 \%$ in females and $41-58 \%$ in males (see Figure 1). The males' rates of imminent re-arrest are higher than that of the females' at ages 14-18 years, but are similar after age 18.

Case managers working with adolescents and
young adults who have serious mental health
conditions can easily identify good candidates
for offending reduction interventions simply by
asking their $15-22$ year old clients if they were
arrested the previous year. Among individuals
reporting an arrest, males have roughly a 50\%
chance of imminent re-arrest, and the females
a $30-40 \%$ chance.

The criminal justice literature suggests that minority race/ethnicity and involvement in specific kinds of illegal behaviors (such as drug misuse) increase risk of persistence in criminal behaviors. Therefore, CMHSR researchers examined whether consideration of these factors contributed to our ability to identify groups at high risk for imminent 
arrest. Interestingly, researchers found no evidence that belonging to any particular racial group increased the likelihood of arrest or re-arrest at any time during the observation period. Similarly, specific offense histories (e.g., a history of a violent crime, or drug crime), considered either cumulatively or in a particular year, did not improve the ability to identify groups at high risk for imminent re-arrest.

Thus, at this point, the best predictor of imminent arrest is a recent arrest. This appears to be generally more true for males compared with females at age 14-18 years and for both genders when the arrest is not their first. Future studies will examine clinical risk factors, such as presence of a substance use disorder and recent mental health service use history.
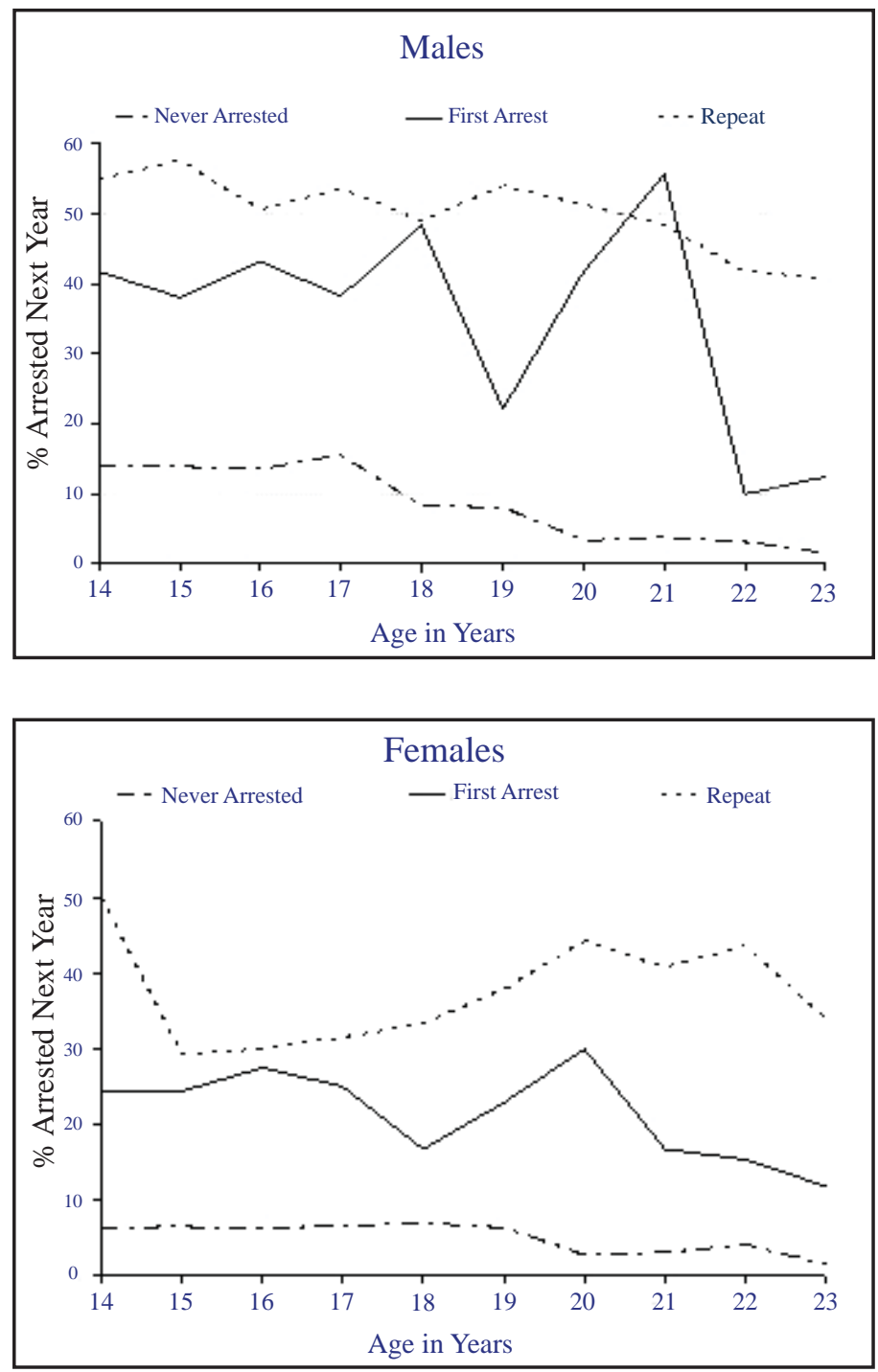

Figure 1. Proportion of youths arrested the following year depending on arrest history at each age; never arrested, arrested for the first time, or a repeat arrest, in a cohort of intensive public adolescent mental health system users.

\section{Recommendations for Policy Makers and Service Providers}

Juvenile or criminal justice involvement can gravely hamper young people's ability to finish school, get a job, and find a place to live. It can also worsen their mental health status and strain family relationships. Both the crimes themselves and resulting justice system involvement impose costs on society with respect to public safety and justice system burden. Therefore, it is important to address these individuals' offending behaviors while they remain mental health service recipients. Toward this end, public mental health systems should:

1. Consider arrest histories of adolescent and young adult clients;

2. Coordinate services between mental health, and juvenile and criminal justice systems;

3. Provide for continuity of services during ages 16-25; and

4. Provide those at high risk with the best services targeted at reducing antisocial behavior.

The following are potentially efficacious treatments for antisocial or disruptive behavior in juveniles: 2

- Functional Family Therapy

- Multisystemic Therapy

- Treatment Foster Care

- Anger Control Training with Stress Inoculation

Approaches with supportive evidence for antisocial behavior in adults include: 3,4

- Social Skills Training

- Social Problem-Solving Training

- Rational Emotive Therapy

- Cognitive Skills Programs

- Relapse Prevention Models

- Aggression Replacement Training

\section{References}

1. Davis, M., Banks, S., Fisher, W., Gershenson, B., \& Grudzinskas, A. (2007). Arrests of adolescent clients of a public mental health system during adolescence and young adulthood. Psychiatric Services, 58, 14541460.

2. Brestan, E., \& Eyberg, S.M.(1998). Effective psychosocial treatments of conduct-disordered children and adolescents: 29 years, 82 studies, and 5,272 kids. Journal of Clinical Child Psychology, 27, 180-189.

3. Lipsey, M.W., Chapman, G., \& Landenberger, N.A. (2001). Cognitivebehavioral programs for offenders. The Annals of the American Academy of Political and Social Science, 578, 144-157.

4. Pearson, F., Lipton, D., Cleland, C., \& Yee, D. (2002). The effects of behavioral/cognitive programs on recidivism. Crime and Delinquency, 48 , 476-496. 